\title{
Accurate Analytical Spiral Inductor Modeling Techniques for Efficient Design Space Exploration
}

\author{
Arthur Nieuwoudt, Student Member, IEEE, Michael S. McCorquodale, Member, IEEE, \\ Ruba T. Borno, Member, IEEE, and Yehia Massoud, Member, IEEE
}

\begin{abstract}
Efficient modeling techniques are required to accelerate design space exploration for integrated spiral inductors. In this letter, closed-form modeling techniques for the inductor's physical inductance and substrate eddy currents are introduced. The model provides several orders of magnitude performance improvement over field-solver-based approaches with typical errors of less than $4 \%$ while demonstrating excellent agreement with measured data from fabricated inductors.
\end{abstract}

Index Terms-Design space exploration, eddy currents, inductance modeling, spiral inductors, substrate modeling.

\section{INTRODUCTION}

D ESIGN SPACE exploration for analog circuits is vital to meet the increasing demand for integrated mixed-signal systems. The design of integrated spiral inductors continues to be an important factor in the development of analog circuits such as low-noise amplifiers, voltage-controlled oscillators, and bandpass filters [1]. Efficient modeling techniques are required to rapidly optimize and synthesize spiral inductor designs [2], [3]. Numerical field solvers most accurately characterize spiral inductors but have large simulation times and memory requirements [4]. While not as accurate as field solvers, closed-form analytical models provide the speed necessary for initial design space exploration. Previously proposed closed-form modeling techniques for inductance either require technology specific fitted parameters [5], [6] or may not provide enough accuracy across the entire design space [7]-[9].

In this letter, we introduce accurate analytical modeling techniques for the physical inductance and substrate eddy currents in spiral inductors. The model provides orders of magnitude performance improvement over numerical field-solver-based approaches with typical errors of less than $4 \%$ and less than $10 \%$ error when compared with fabricated inductor data.

\section{Proposed Inductor Modeling Techniques}

\section{A. Analytical Inductance Model}

To calculate the physical inductance of the inductor, we developed a formulation that exploits the inductor's symmetry to significantly reduce computational cost over approaches based

Manuscript received June 27, 2006; revised October 2, 2006. The review of this letter was arranged by Editor A. Wang.

A. Nieuwoudt and Y. Massoud are with Rice University, Houston, TX 77005 USA (e-mail: massoud@ rice.edu).

M. S. McCorquodale and R. T. Borno are with Mobius Microsystems, Inc., Detroit, MI 48226 USA.

Digital Object Identifier 10.1109/LED.2006.886329

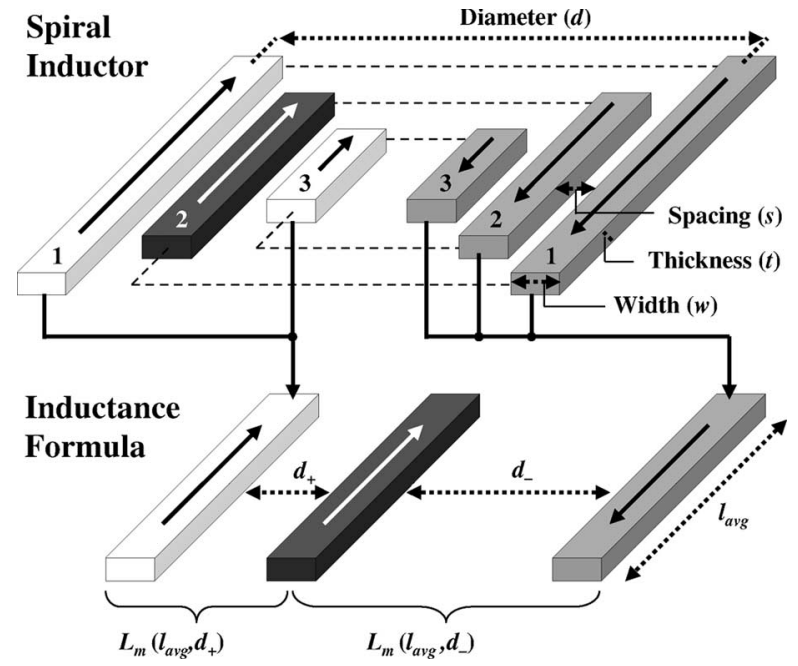

Fig. 1. Inductor design parameters include turns $(n)$, inductor diameter $(d)$, conductor width $(w)$, conductor spacing $(s)$, and conductor thickness $(t)$. The physical inductance model depends on the interaction between two pairs of virtual conductors.

on field solvers. The physical inductance can be expressed as the sum of self- and mutual-inductances, which can be calculated using [8]. We approximate the average mutual inductance between a given conductor and conductors on both adjacent and opposite sides of the inductor, as depicted in Fig. 1. The mutual inductance depends on the weighted average length and distance between conductors with current flowing in the same direction $\left(l_{+}\right.$and $\left.d_{+}\right)$and in opposite directions $\left(l_{-}\right.$and $\left.d_{-}\right)$. The average length between all combinations of conductors on the same side of the inductor is

$$
l_{+}=\frac{1}{n^{2}-n} \sum_{\substack{i, j \\ i \neq j}}^{n}\left(\frac{l_{i}+l_{j}}{2}\right)=d-\frac{(n-1)^{2} p}{n}
$$

where $l_{i}$ and $l_{j}$ are the lengths of the longest conductors on the $i$ th and $j$ th turns of the inductor, and $p=w+s$, as depicted in Fig. 1. The average length between pairs of conductors on opposite sides of the inductor is

$$
l_{-}=\frac{1}{n^{2}} \sum_{i, j}^{n}\left(\frac{l_{i}+l_{j}}{2}\right) \text {. }
$$

Expanding the sum in (2) reveals that $l_{-}=l_{+}$, which we refer to as $l_{\text {avg }}$ for the remainder of this letter. 
The average distance between pairs of conductors with currents flowing in the same direction can be expressed as

$$
d_{+}=\frac{1}{l_{\text {avg }}\left(n^{2}-n\right)} \sum_{\substack{i, j \\ i \neq j}}^{n}\left(\frac{l_{i}+l_{j}}{2} d_{+(i, j)}\right)
$$

where $d_{+}$is the distance between the conductors in the $i$ th and $j$ th turns on the same side of the inductor. By expanding the sum in (3), $d_{+}$is simplified to yield

$$
d_{+}=\frac{p(2 d(n+1)+p-2 n p(n-1)}{6 l_{\mathrm{avg}}} .
$$

Similarly, the average distance between pairs of conductors on opposite sides of the conductor can be expressed as

$$
d_{-}=\frac{1}{l_{\text {avg }} n^{2}} \sum_{i, j}^{n}\left(\frac{l_{i}+l_{j}}{2} d_{-(i, j)}\right)
$$

where $d_{-}$is the distance between the conductors in the inductor's $i$ th and $j$ th turns on opposite sides of the inductor. Expanding the summation yields

$$
\begin{aligned}
d_{-}=\frac{6 d^{2} n-6 d p(n-1)(2 n-1)}{6 n l_{\text {avg }}} & \\
& +\frac{p^{2}(n-1)(3+n(7 n-11))}{6 n l_{\text {avg }}} .
\end{aligned}
$$

Using the average length $\left(l_{\mathrm{avg}}\right)$ and distance of the conductors on the same $\left(d_{+}\right)$and opposite $\left(d_{-}\right)$sides of the inductor, the overall mutual inductance is

$$
L_{\mathrm{mut}}=4\left(\left(n^{2}-n\right) L_{m}\left(l_{\mathrm{avg}}, d_{+}\right)-n^{2} L_{m}\left(l_{\mathrm{avg}}, d_{-}\right)\right)
$$

where $L_{m}(l, d)$ can be calculated using the mutual inductance formula in [8]. For a fractional number of turns, we interpolate between the inductance results for $n=\lfloor n\rfloor$ and $n=\lfloor n\rfloor+1$. Note that inductance formulation is physics-based and can be extended to octagonal or circular geometries.

\section{B. Substrate-Eddy-Current Model}

Spiral inductors fabricated on highly conductive substrates experience significant losses due to the magnetically induced eddy currents. The model utilizes complex-image theory to capture substrate eddy currents, where the substrate can be replaced with a mirror image of the spiral inductor at a complex depth. To determine the necessary complex depth, consider an infinitely long conductor that lies at a height $z_{o}$ above the substrate. The electric field in the region between the substrate and the conductor can be expressed as

$$
E=\frac{-j \mu_{o} w I}{2 \pi} \int_{0}^{\infty} \frac{1}{\lambda}\left(e^{-\lambda\left(z-z_{o}\right)}+R(\lambda) e^{-\lambda\left(z+z_{o}\right)}\right) \cos (\lambda x) d \lambda
$$

where $I$ is the current in the conductor, $\omega$ is the frequency $(\mathrm{rad} / \mathrm{s}), z_{0}$ is the height of the conductors above the substrate, and $R(\lambda)$ is the substrate reflection coefficient [10], [11].
In order to model the contribution of the substrate to the electric field with an image conductor, $R(\lambda)$ in (8) can be replaced with $R(\lambda)=f(\lambda) e^{-\alpha \lambda} \longrightarrow f(\lambda)=R(\lambda) e^{\alpha \lambda}$, where $f(\lambda)$ is an unknown function. Since $f(\lambda)$ is an exponential function, $d f / d \lambda=f(\lambda) g(\lambda, \alpha)$, where $g(\lambda, \alpha)$ is a function of the argument in the exponential of $f(\lambda)$. The compleximage depth, $\alpha$, is then determined by solving the equation $g(\lambda, \alpha)=0$ and using the low-order terms in the Taylor series expansion of $f(\lambda)$. We have verified the expression for the complex-image depth for a single-layer floating substrate presented in [11] and derived the following explicit expressions for the complex-image depths for 2- and 3-layer floating substrates:

$$
\begin{aligned}
\alpha_{2} & =\frac{2\left(\gamma_{1}+\gamma_{2} A B\right)}{\gamma_{1}\left(\gamma_{1} A+\gamma_{2} B\right)} \\
\alpha_{3} & =\frac{2 \gamma_{2} A\left(\gamma_{2} B+\gamma_{3} C\right)+2 \gamma_{1}\left(\gamma_{2}+\gamma_{3} B C\right)}{\gamma_{1} \gamma_{2}\left(\gamma_{1} A+\gamma_{2} B\right)+\gamma_{1} \gamma_{3}\left(\gamma_{2}+\gamma_{1} A B C\right)} \\
A & =\tanh \left(\gamma_{1} h_{1}\right), \quad B=\tanh \left(\gamma_{2} h_{2}\right), \quad C=\tanh \left(\gamma_{3} h_{3}\right) \\
\gamma_{1} & =\frac{1+j}{\delta_{1}}, \quad \gamma_{2}=\frac{1+j}{\delta_{2}}, \quad \gamma_{3}=\frac{1+j}{\delta_{3}}
\end{aligned}
$$

where $h_{1}, \delta_{1}, h_{2}, \delta_{2}, h_{3}$, and $\delta_{3}$ are the heights and skin depths of substrate layers 1,2 , and 3 , respectively, where layer 1 is the top layer of the substrate. Once the complex-image depth is known, substrate eddy currents can be modeled by placing a mirror image of the inductor at a complex distance $D=2 t_{\mathrm{ox}}+\alpha$, where $t_{\mathrm{ox}}$ is the height of the inductor above the substrate. The impact of substrate eddy currents on the inductor is then captured using $L_{\text {eddy }}=L_{\text {image }}(\operatorname{Re}(D))$ and $R_{\text {eddy }}=$ $-\omega \operatorname{Im}\left(L_{\text {image }}(D)\right)$, where $L_{\text {image }}$ is the mutual inductance between each segment of the inductor and the image conductors separated by a complex distance $D$.

\section{RESULTS}

We compared the analytical model results with both current closed-form inductance modeling techniques and simulated results. The model is also compared with measurements taken from inductors fabricated in a $0.18-\mu \mathrm{m} \mathrm{RF-CMOS} \mathrm{process.} \mathrm{For}$ quality factor and effective inductance calculations, the analytical physical inductance and eddy-current models are used in a frequency-dependent eleven-element $\pi$ model [3]. Capacitive effects due to substrate leakage currents are modeled using [12]. We capture high-frequency resistive effects in the conductors, including skin effect, using the modeling techniques described in [13]. The series inductance model is compared with the field solver, FastHenry, for numerous inductor designs with typical geometric dimensions and operating frequencies, which are listed in Table I. Our method has an average error of $2.5 \%$ with $99 \%$ of the cases having less than $6.0 \%$ error. When compared with the inductor designs simulated in FastHenry, the expressions in [9] and [7] have mean errors of $6.5 \%$ and $2.8 \%$ and with $99 \%$ of the cases having less than $10.5 \%$ and $15.0 \%$, respectively.

To evaluate the accuracy and performance of the eddycurrent formulation, 650 spiral inductor designs were simulated over highly conductive one-, two-, or three-layer substrates with 
TABLE I

DESIGN PARAMETERS FOR INDUCTOR SIMULATIONS

\begin{tabular}{|c|c|c|}
\hline Design Parameter & Minimum & Maximum \\
\hline \hline Number of Turns & 2 & 8 \\
\hline Conductor Width $(\mu m)$ & 1 & 20 \\
\hline Conductor Spacing $(\mu m)$ & 0.5 & 3 \\
\hline Conductor Thickness $(\mu m)$ & 0.5 & 3 \\
\hline Inductor Diameter $(\mu m)$ & 50 & 400 \\
\hline Substrate Layers & 1 & 3 \\
\hline Oxide Thickness - $t_{o x}(\mu m)$ & 4 & 10 \\
\hline Layer 1 Thickness - $h_{1}(\mu m)$ & 4 & 10 \\
\hline Layer 2 Thickness - $h_{2}(\mu m)$ & 4 & 10 \\
\hline Layer 3 Thickness - $h_{3}(\mu m)$ & 500 & 500 \\
\hline Layer 1 Conductivity - $\sigma_{1}(\mu m \cdot \Omega)^{-1}$ & $10^{-5}$ & 0.005 \\
\hline Layer 2 Conductivity - $\sigma_{2}(\mu m \cdot \Omega)^{-1}$ & $10^{-5}$ & 0.005 \\
\hline Layer 3 Conductivity - $\sigma_{3}(\mu m \cdot \Omega)^{-1}$ & $10^{-5}$ & 0.005 \\
\hline Operating Frequency $(\mathrm{GHz})$ & 1 & 5 \\
\hline
\end{tabular}

the process parameters listed in Table I using both compleximage theory and the field solver. For the effective inductance contributed by substrate eddy currents, the average error produced by the analytical model was $0.1 \%$ with a maximum error of $0.6 \%$ while the average error for the effective resistance was $3.9 \%$ with $93 \%$ of the cases experiencing less than a $10 \%$ error. Cases with a thin highly conductive top substrate layer and a low-conductivity bulk layer experienced the most error from the truncated higher order terms in the Taylor series approximation of $f(\lambda)$.

For physical validation of the modeling techniques, two square hollow-core inductors were fabricated in a $0.18-\mu \mathrm{m}$ RF-CMOS process with a $2-\mu$ m-thick top metal layer. A die micrograph is shown in Fig. 2. Test measurements were acquired by probing the fabricated die using a microwave probe station and $40-\mathrm{GHz}$ air coplanar probes with a $100-\mu \mathrm{m}$ pitch ground-signal ground configuration (ACP-40GSG-100). Fig. 3 displays the measured and simulated quality factor $\operatorname{Im}\left(Z_{11}\right) / \operatorname{Re}\left(Z_{11}\right)$ and effective inductance $\operatorname{Im}\left(Z_{11}\right) / \omega$ for each inductor, where $Z_{11}$ is the impedance parameter of the inductor, and $\omega$ is the operating frequency in radians per second. For the $6 \mathrm{nH}$ inductor, the mean error for the quality factor was $4.1 \%$, while the mean error for the effective inductance was $3.8 \%$. Similarly, for the $8 \mathrm{nH}$ inductor, the mean quality factor error was $9.9 \%$, and the mean effective inductance error was $7.5 \%$. Our spiral inductor model simulates 1601 frequency points in Fig. 3 in approximately $1 \mathrm{~s}$ for both inductors, while the field solver, FastHenry [4], requires $40000 \mathrm{~s}$ for inductance and resistance extraction.

\section{CONCLUSION}

In this letter, we present closed-form modeling techniques for physical inductance and substrate eddy currents in spiral inductors. The model agrees with field-solver data and measured results from fabricated inductors with orders of magnitude improvement in computational performance. The proposed

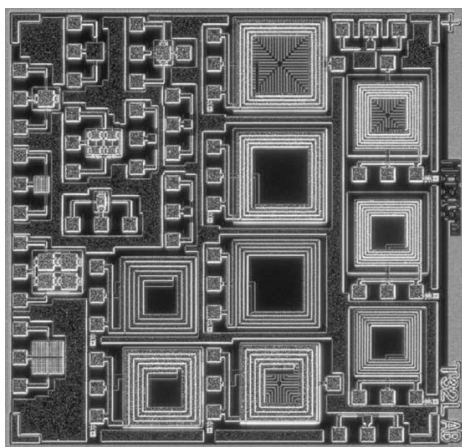

Fig. 2. Die micrograph of the $0.18-\mu \mathrm{m}$ inductor test chip.

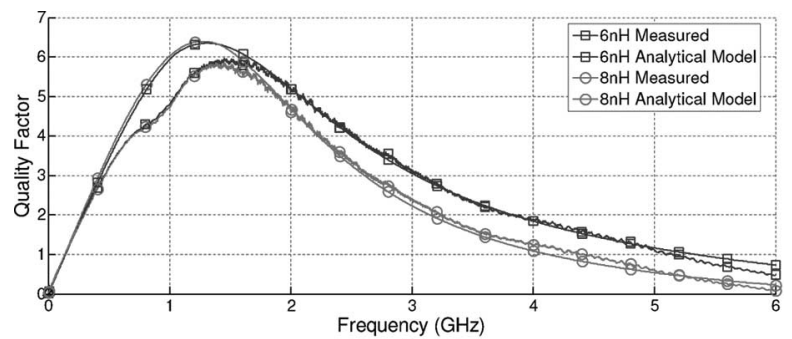

(a)

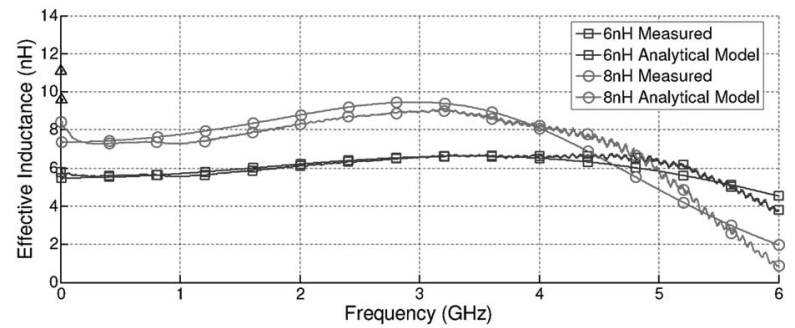

(b)

Fig. 3. (a) Quality factor and (b) effective inductance for the fabricated inductors and the model. (Color version available online at http:// ieeexplore.ieee.org.)

model will provide designers and design-automation tools with the means to rapidly explore the inductor design space to meet the demands of tomorrow's mixed-signal systems.

\section{REFERENCES}

[1] A. Nieuwoudt, T. Ragheb, and Y. Massoud, "SOC-NLNA: Synthesis and optimization for fully integrated narrow-band CMOS low noise amplifiers," in Proc. IEEE/ACM DAC, Jul. 2006, pp. 879-884.

[2] A. Nieuwoudt and Y. Massoud, "Multi-level approach for integrated spiral inductor optimization," in Proc. IEEE/ACM DAC, Jun. 2005, pp. 648-651.

[3] — , "Robust automated synthesis methodology for integrated spiral inductors with variability," in Proc. IEEE/ACM Int. Conf. Comput.-Aided Des., Nov. 2005, pp. 502-507.

[4] M. Kamon, M. J. Tsuk, and J. White, "FastHenry: A multipole-accelerated 3-D inductance extraction program," IEEE Trans. Microw. Theory Tech., vol. 42, no. 9, pp. 1750-1758, Sep. 1994

[5] J. Sieiro, J. Lopez-Villegas, J. Cabanillas, J. Osorio, and J. Samitier, "A physical frequency-dependent compact model for RF integrated inductors," IEEE Trans. Microw. Theory Tech., vol. 50, no. 1, pp. 384-392, Jan. 2002.

[6] A. Watson, D. Melendy, P. Francis, K. Hwang, and A. Weisshaar, "A comprehensive compact-modeling methodology for spiral inductors in silicon-based RFICs," IEEE Trans. Microw. Theory Tech., vol. 52, no. 3, pp. 849-857, Mar. 2004. 
[7] S. Mohan, M. del Mar Hershenson, S. Boyd, and T. Lee, "Simple accurate expressions for planar spiral inductances," IEEE J. Solid-State Circuits, vol. 34, no. 10, pp. 1419-1424, Oct. 1999.

[8] C. Yue and S. Wong, "Physical modeling of spiral inductors on silicon," IEEE Trans. Electron Devices, vol. 47, no. 3, pp. 560-568, Mar. 2000.

[9] S. Jenei, B. Nauwelaers, and S. Decoutere, "Physics-based closed-form inductance expression for compact modeling of integrated spiral inductors," IEEE J. Solid-State Circuits, vol. 37, no. 1, pp. 579-586, Jan. 2002.

[10] J. R. Wait and K. P. Spies, "On the image representation of the quasi-static fields of a line current source above the ground," Can. J. Phys., vol. 47, no. 23, pp. 2731-2733, 1969.
[11] A. Weisshaar and A. Luoh, "Closed-form expressions for the series impedance parameters of on-chip interconnects on multilayer silicon substrates," IEEE Trans. Adv. Packag., vol. 27, no. 1, pp. 126-134, Feb. 2004.

[12] Y. Cao, R. Groves, X. Huang, N. Zamdmer, J. Plouchart, R. Wachnik, T. King, and C. Hu, "Frequency-independent equivalent-circuit model for on-chip spiral inductors," IEEE J. Solid-State Circuits, vol. 38, no. 3, pp. 419-426, Mar. 2003.

[13] A. Nieuwoudt and Y. Massoud, "Analytical wide-band modeling of high frequency resistance in integrated spiral inductors," Analog Integrated Circuits and Signal Processing: Mixed Signal Letters, 2006, in press. 